\title{
1 A brain atlas of the camouflaging dwarf cuttlefish, Sepia bandensis
}

2

3

4

5

6

34 The coleoid cephalopods are a group of soft-bodied marine mollusks that exhibit an array of interesting

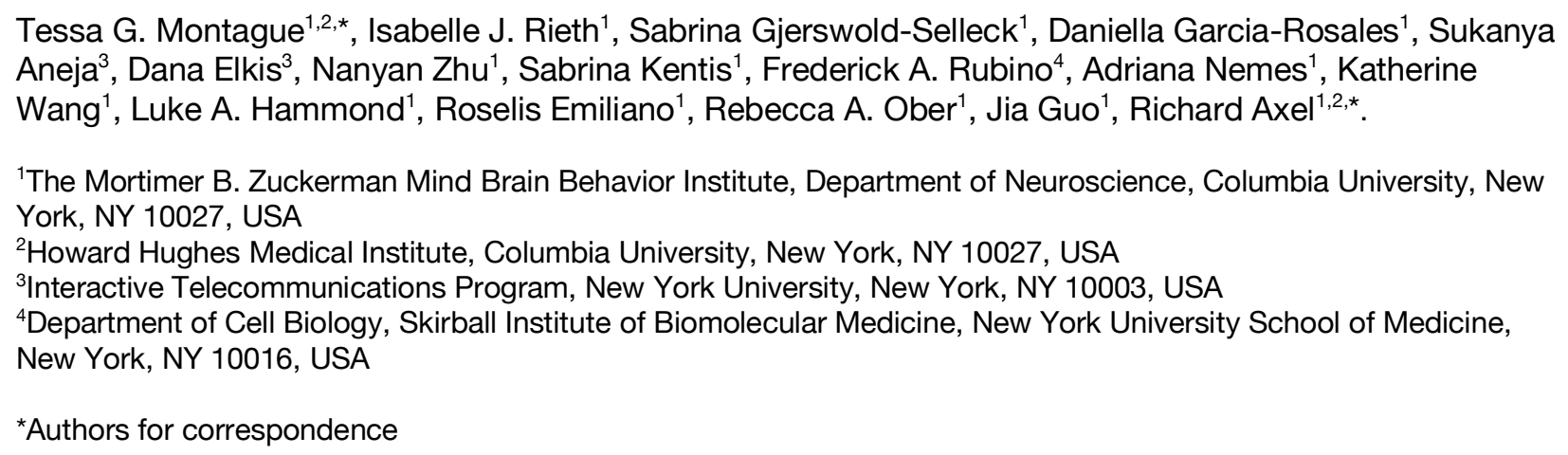
Aneja $^{3}$, Dana Elkis ${ }^{3}$, Nanyan Zhu' ${ }^{1}$, Sabrina Kentis ${ }^{1}$, Frederick A. Rubino ${ }^{4}$, Adriana Nemes ${ }^{1}$, Katherine Wang $^{1}$, Luke A. Hammond ${ }^{1}$, Roselis Emiliano ${ }^{1}$, Rebecca A. Ober ${ }^{1}$, Jia Guo ${ }^{1}$, Richard Axel ${ }^{1,2, *}$.

${ }^{1}$ The Mortimer B. Zuckerman Mind Brain Behavior Institute, Department of Neuroscience, Columbia University, New York, NY 10027, USA

${ }^{2}$ Howard Hughes Medical Institute, Columbia University, New York, NY 10027, USA

${ }^{3}$ Interactive Telecommunications Program, New York University, New York, NY 10003, USA

${ }^{4}$ Department of Cell Biology, Skirball Institute of Biomolecular Medicine, New York University School of Medicine, New York, NY 10016, USA

*Authors for correspondence

\section{Abstract}

The dwarf cuttlefish, Sepia bandensis, a small cephalopod that exhibits dynamic camouflage, is an emerging model organism in neuroscience. Coleoid cephalopods (cuttlefish, octopus, and squid) evolved large, complex brains capable of learning, problem-solving, and memory. We used highresolution magnetic resonance imaging (MRI), deep learning, and fluorescent histology to generate a dwarf cuttlefish brain atlas and built an interactive web tool (cuttlebase.org) to host the data. Guided by observations in other cephalopods, we identified 38 brain lobes. The dwarf cuttlefish brain is partially encased in cartilage and includes two large optic lobes (74\% the total volume of the brain), chromatophore lobes whose motor neurons directly innervate the skin, and a vertical lobe that has been implicated in learning and memory. Motor neurons emerging from the chromatophore lobe modulate the color, pattern, and texture of the skin to elicit camouflage. This brain atlas provides a valuable tool for exploring the neural basis of cuttlefish behavior.

\section{Introduction} biological phenomena, including three hearts, blue blood, color-changing skin, prehensile regenerating 
36 arms, and elaborate motor and social behaviors. In contrast to ancient cephalopods, which possess

37 stereotypical molluskan shells, the coleoid cephalopods internalized or lost these shells over

38 evolutionary time. In addition, they evolved larger brains, camera-type eyes and neurally controlled

39 changes in skin pattern and texture for camouflage and social communication (Amodio et al., 2019;

40 Hanlon \& Messenger, 2018). During camouflage, cuttlefish create an approximation of the physical world

41 on their skin. Camouflage is optically driven (Young, 1971; Hanlon \& Messenger, 1988) and is a

42 consequence of the activation of motor neurons that project from the brain to radial muscles that

43 surround pigment-filled saccules (chromatophores) of the skin. Excitation elicits an expansion of the

44 chromatophore to reveal pixels of different colors (Messenger, 2001).

46 The coleoid cephalopod brain surrounds the esophagus and investigators have divided the brain into

47 supra- and subesophageal structures (Dietl, 1878; Hillig, 1912). The supraesophageal structures include

48 a vertical lobe complex involved in learning and memory, and a supraesophageal mass involved in the

49 coordination of motor behaviors. The subesophageal mass comprises multiple motor areas that elicit

50 simpler motor actions (Boycott \& Young, 1955; Boycott, 1961; Sanders \& Young, 1940). The optic lobe

51 complex, the largest brain structure, resides lateral to the central peri-esophageal structures, and is

52 engaged in visual processing (Young, 1974; Young, 1976; Young, 1977; Young, 1979). The connectivity

53 and function of these structures have been studied by histology (Young, 1971; Young, 1974; Young,

54 1976; Young, 1977; Young, 1979; Messenger, 1979), tracing (Saidel, 1982; Robertson et al., 1993;

55 Dubas et al., 1986b; Gaston \& Tublitz, 2004; Gaston \& Tublitz, 2006), lesion (Wells \& Young, 1975;

56 Boycott \& Young, 1957; Chichery \& Chichery, 1987; Fiorito \& Chichery, 1995; Messenger, 1967;

57 Graindorge et al., 2006), magnetic resonance imaging (Chung et al., 2020; Chung et al., 2021) and

58 electrophysiology (Chichery \& Chanelet, 1976; Boycott, 1961; Dubas et al., 1986a; Bullock \& Budelmann,

59 1991; Zullo et al., 2009; Hochner et al., 2003; Shomrat et al., 2011). Systematic stimulation of individual

60 brain lobes of the European cuttlefish (Sepia officinalis) revealed their participation in different motor

61 behaviors, including the movement of the fins, arms and chromatophores (Boycott, 1961). The function

62 of higher processing centers in the supraesophageal mass, however, remain elusive. 
64 The European cuttlefish, Sepia officinalis, has been studied most extensively to understand behavior

65 (Hanlon \& Messenger, 2018), learning and memory (Turchetti-Maia et al., 2019) and the neural control of

66 skin patterning (Reiter et al., 2018; Hanlon, 2007). We study the dwarf cuttlefish, Sepia bandensis, a

67 tropical species from the Indo-Pacific (Figure 1A-D, Video 1). Dwarf cuttlefish are small $(<6 \mathrm{~cm}$ mantle

68 length), embryonic development is relatively fast (1 month) and they reach sexual maturity in only 4

69 months (Montague et al., 2021). Dwarf cuttlefish can be bred at relatively high density in the lab, each

70 animal produces dozens of embryos over its lifetime, and the embryos can be cultured in vitro to

71 hatching (Montague et al., 2021). These features may permit the introduction of genetically-encoded

72 calcium indicators and light-activated channels that may facilitate an understanding of the relationship

73 between neural activity and behavior.

75 The study of the neural basis of skin patterning and behavior in the dwarf cuttlefish requires knowledge

76 of its neuroanatomy. Anatomical mapping using histology or MRI has been performed in a small number

77 of octopus and squid species (Chung et al., 2020; Koizumi et al., 2016; Jung et al., 2018; Chung et al.,

78 2021), but a brain atlas has not been generated for any cuttlefish species. In this study, we used high-

79 resolution MRI and deep learning to build a 3D model of the dwarf cuttlefish brain, which we annotated

80 with 38 lobes and nine nerve tracts. In addition, we performed three-plane, whole brain fluorescence

81 histology of the brain, and we used MRI to create an anatomical model of an entire cuttlefish, including

82 the animal's reproductive, digestive, respiratory and circulatory systems. These data allowed us to

83 create an interactive, user-friendly web tool, Cuttlebase (cuttlebase.org).

\section{Results}


90 The cuttlefish brain is located posterior and medial to the eyes and is encased posteriorly by cranial

91 cartilage (Figure 1E). We performed ex vivo magnetic resonance imaging (MRI) of 8 adult dwarf cuttlefish

92 brains (4 males, 4 females) at $50 \mu \mathrm{m}$ isotropic resolution. Deep learning techniques were applied to

93 extract the brains from their surrounding tissues (see Methods, Figure S1). We used prior

94 neuroanatomical descriptions to guide the annotation of brain lobes (Boycott, 1961; Chung et al., 2020).

95 Finally, we co-registered the 8 brains to create a merged, annotated template brain (Figure 1F). The ex

96 vivo dwarf cuttlefish brain is $95 \%$ the volume of the ex vivo mouse brain (see Methods).

98 The dwarf cuttlefish brain can be divided into 38 discrete lobes. This value is in accord with previous

99 anatomical studies in other species, but it remains possible that the annotated lobes can be subdivided

100 further. The largest brain lobes, the optic lobes, comprise $74 \%$ of the volume of the brain (Table 2) and

101 receive direct projections from the retina. Camouflage is initiated by visual information represented in the

102 optic lobe, which projects directly to the lateral basal lobe (Young, 1962; Boycott, 1961; Young, 1971;

103 Young, 1974). The lateral basal lobe projects to the anterior and posterior chromatophore lobes, which

104 control the chromatophores on the head and arms, and the mantle, respectively (Boycott, 1961; Young,

105 1976). The posterior chromatophore lobe projects motor neurons to the mantle skin via the pallial nerve,

106 which controls chromatophores and papillae to create skin patterns and three-dimensional texture

107 (Gonzalez-Bellido et al., 2018; Young, 1972; Messenger, 2001; Boycott, 1961). We have focused on the

108 flow of information thought to elicit skin patterning, but the atlas provides information on the brain

109 regions involved in several additional behaviors (Table 1) (Nixon \& Young, 2003; Hanlon \& Messenger,

110 2018).

\section{A histological brain atlas for the dwarf cuttlefish}

114 We complemented the anatomical description of the cuttlefish brain with histological examination of the

115 entire brain to obtain cellular resolution. We sectioned the brain in the transverse, horizontal and sagittal

116 planes (Figure 2A). All sections were stained with Phalloidin, an F-actin peptide that labels axons, and 

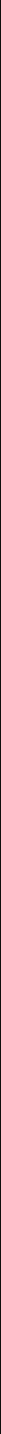

Figure 2. Histological brain atlas of the dwarf cuttlefish, Sepia bandensis. A) The cuttlefish brain can be sectioned in the transverse, horizontal and sagittal planes. B) Histological slices of the cuttlefish brain stained with NeuroTrace, a Nissl stain that labels cell bodies, and annotated with 38 brain lobes and 9 nerve tracts. Scale bar, 1 mm. For abbreviations see Table 1. C) Confocal image of the layered cortex of the optic lobe (white square in Figure 2B). Scale bar, $100 \mu \mathrm{m}$. 
117 NeuroTrace, a Nissl stain that labels cell bodies. Our annotated 3D MRI datasets and a prior

118 neuroanatomical description (Boycott, 1961) were used to describe the histological organization of 38

119 brain lobes and 9 nerve tracts (Figure 2B). Phalloidin and NeuroTrace staining confirmed organizational

120 features of the cephalopod brain. The majority of lobes are discrete and bounded, a feature not

121 uniformly observed in vertebrate brains. Each lobe contains an outer perikaryal layer of cell bodies that

122 surrounds a dense, inner neuropil (Figure 2B). The optic lobe is comprised of a layered cortex, with two

123 cell layers apposing a plexiform zone of fibers (Young, 1962; Young, 1974) (Figure 2C) and a central

124 medulla with cell islands that are connected in a tree-like structure (Liu et al., 2017; Young, 1962; Young,

125 1974). The two outer layers may share properties with the lamina and medulla of dipteran optic lobes

126 (Shinomiya et al., 2019).

128 Cuttlebase - a web tool for visualizing the cuttlefish brain

130 We built an interactive web tool to maximize the utility of our brain atlas (Figure 3A). Cuttlebase features

131 multiple tools for the dwarf cuttlefish, including 3D models of the adult cuttlefish brain (Figure 3B) and

132 body (Figure 3C), as well as annotated histological sections of the brain in 3 planes (Figure 3A). We

133 visualized the cranial cartilage - the only rigid tissue around the brain - in the 3D and histological atlas

134 to facilitate the development of methods to head-fix cuttlefish, an important step for stabilizing the brain

135 for two-photon imaging or electrophysiology. Cuttlebase is easy-to-use, with an array of features

136 designed for both novice and expert users, including responsive, color-coded labels for each brain

137 region, the ability to zoom, rotate and screenshot the data, synchronized graphics that denote the

138 brain's orientation, and options to show the brain's position within the cuttlefish body.

\section{Discussion}


143 The brain of the dwarf cuttlefish exhibits structural similarities with other decapodiformes, the 10-armed

144 cuttlefish and squid, but is somewhat different from the brain of octopodiformes, octopus and vampire

145 squid (Albertin \& Simakov, 2020). First, the decapodiformes, but not octopodiformes, possess a giant-

146 fiber system, which is used for funnel-based jet propulsion during escape behaviors. The giant cells are

147 located in the ventral magnocellular lobe, and receive input from the optic lobes and statocysts (the

148 cephalopod vestibular system) (Nixon \& Young, 2003; Abbott et al., 1995). Second, the brachial, pedal

149 and inferior frontal lobes of octopus are larger than the decapodiformes, which may facilitate their more

150 sophisticated use of arms and tactile learning (Ponte et al., 2020). Third, the octopus vertical lobe is

151 folded into gyri, creating a larger surface area (Young, 1971). Finally, the decapodiformes, but not

152 octopodiformes, possess a fin lobe, used for fin locomotion (Boycott, 1961). Most decapodiformes use

153 two forms of locomotion: jet propulsion using the funnel, and swimming using the funnel and fins

154 (Russell \& Steven, 1930). Interestingly, the dwarf cuttlefish uses an additional mode: it can walk bipedally

155 using its ventral arms (Video 2). This may require the evolution of a control system for coordinated

156 locomotion.

158 Analysis of the dwarf cuttlefish brain atlas reveals notable differences between the brains of the coleoid

159 cephalopods (octopus, cuttlefish and squid) and the nautilus, a living order of an otherwise extinct

160 lineage (Crook \& Basil, 2008). Unlike the large, centralized brains of the coleoid cephalopods, the

161 nautilus central nervous system features 3 simple nerve cords with minimal subdivisions. The nautilus

162 brain does not appear to contain the motor areas for the elaborate control of limbs, nor the higher brain

163 areas for learning and memory (Young, 1965; Budelmann, 1995). Nautiluses have pinhole eyes (Muntz \&

164 Raj, 1984) and smaller optic lobes (Young, 1965). However, the olfactory lobes of nautiluses are much

165 larger than that of the coleoid cephalopods (Young, 1965), consistent with the central role of olfaction in

166 nautilus foraging (Basil et al., 2000).

168 The dwarf cuttlefish brain atlas presented here serves multiple functions. First, the MRI-based 3D brain

169 model affords users the ability to locate specific targets for electrophysiological recordings or calcium 
170 imaging experiments of neural activity. Second, the histological atlas facilitates the identification of brain

171 regions in anterograde and retrograde tracing experiments. Third, the neuroanatomical and histological

172 atlases facilitate the identification of regionally-restricted genes that may provide specificity to efforts at

173 genetic manipulation. Fourth, the entire dataset is a comprehensive resource for comparative

174 neuroanatomical analyses. Finally, the addition of a cuttlefish body atlas allows users to see the

175 physiological context of the brain and may permit the study of communication between the brain and

176 internal organs. The generation of the neuroanatomical and histological atlas for the dwarf cuttlefish may

177 inform studies in other cephalopods, for which atlases do not exist, and now renders Sepia bandensis a

178 more facile system for the study of the neural control of camouflage.

\section{$181 \quad$ Materials \& Methods}

\section{$\underline{3 \mathrm{D} \text { brain atlas }}$}

\section{MRI acquisition}

1868 adult cuttlefish (4 male, 4 female) were euthanized in $10 \%$ ethanol, and the brain and connected eyes 187 were surgically removed and fixed overnight in a solution of $4 \%$ paraformaldehyde (PFA) in filtered artificial 188 seawater (FASW) at $4^{\circ} \mathrm{C}$. The fixed brains were washed in PBS, incubated in $0.2 \%$ OMNISCAN 189 (gadodiamide) for 2 days at $4^{\circ} \mathrm{C}$ to increase contrast, and then suspended in fomblin in a $15 \mathrm{~mL}$ conical 190 tube. Imaging was performed on a Bruker BioSpec 94/30 horizontal small animal MRI scanner (field 191 strength, 9.4 T; bore size, $30 \mathrm{~cm}$ ) equipped with a CryoProbe and ParaVision 6.0.1 software (Bruker). A 23

$192 \mathrm{~mm} \mathrm{1H}$ circularly polarized transmit/receive-capable mouse head volume coil was used for imaging. For 193 each cuttlefish, one scan was acquired of the brain and eyes (to obtain a scan of the entire cranial 194 cartilage), and a higher resolution scan was acquired of the brain only. T1-weighted images were acquired 195 with a Fast Low Angle Shot (FLASH) sequence (brain/eye scan: TR $=50 \mathrm{~ms}$, TE $=10 \mathrm{~ms}$, FOV $=30 \times 24 \mathrm{x}$ 
$19615 \mathrm{~mm}^{3}$, voxel size $=100 \times 100 \times 100 \mu \mathrm{m}^{3}$, scan time $=33 \mathrm{~m} 12 \mathrm{~s}$; brain-only scan: $\mathrm{TR}=50 \mathrm{~ms}$, TE $=8.5$

$197 \mathrm{~ms}, \mathrm{FOV}=14 \times 12 \times 11 \mathrm{~mm}^{3}$, voxel size $=60 \times 60 \times 60 \mu \mathrm{m}^{3}$, scan time $=4 \mathrm{hr} 28 \mathrm{~m}$ ).

\section{MRI processing \& brain extraction}

200 All scans underwent N4 bias field correction (Tustison et al., 2010). Whole brain scans were isotropically 201 upsampled to $50 \mu \mathrm{m}$ isotropic resolution with cubic B-spline interpolation. To computationally extract the 202 brains from their surrounding tissue, brain masks were generated by an in-house deep learning model 203 (Gjerswold-Selleck et al., 2021), which was pre-trained with brain masks manually annotated in 3D Slicer 204 (Fedorov et al., 2012). The deep learning brain masks were manually polished using the 3D Slicer Segment 205 Editor and then used to extract the brain from each brain-only MRI scan ("brain-extracted images") (Figure 206 S1). For the brain/eye scans, the cranial cartilage was manually segmented in 3D Slicer using the Segment 207 Editor. The cartilage masks were used to extract the cartilage from each brain/eye MRI scan ("cartilage208 extracted images").

\section{Segmentation}

211 Two of the whole brain scans (1 male, 1 female) were manually segmented in 3D Slicer by 6 independent 212 annotators guided by prior neuroanatomical descriptions (Boycott, 1961; Chung et al., 2020). The brain 213 label maps for each subject were merged using pixel-level majority voting, transformed to the remaining 2146 subjects, and then manually corrected, resulting in 8 brain label maps corresponding to the 8 subjects.

\section{Generation of the template brain}

217 The final MRI atlas was built by merging the brain template, built from the high-resolution brain-only scans 218 with the cranial cartilage template, built from the brain/eye scans. First, to generate each template, a 219 population average of brain-extracted or cartilage-extracted images was constructed through an iterative 220 process by averaging the co-registered images over multiple cycles using a symmetric diffeomorphic 221 algorithm with cubic-spline interpolation (Avants et al., 2011). The brain label map (annotations) for each 222 subject was diffeomorphically transformed to the whole brain template space and combined through pixel- 
223 level majority voting. The brain/eye template was isotropically upsampled to match the $50 \mu \mathrm{m}$ resolution

224 of the whole brain scans, and the whole brain template underwent rigid registration to align it with the

225 brain/eye template. Finally, the brain regions of the whole brain template were combined with the cartilage

226 regions of the whole head template to build a single atlas. In the regions where the two templates

227 overlapped, pixel values of the high-resolution whole brain template were selected. The cuttlefish brain

228 label map was smoothed by taking a majority vote in a local neighborhood with a $3 \times 3 \times 3$ kernel size.

230 Brain volume calculation

231 A mouse MRI brain model (15 $\mu \mathrm{m}$ resolution, average of 18 ex vivo subjects) was downloaded from the

232 Australian Mouse Brain Mapping Consortium (nonsymmetric version, NiFTI format,

233 https://imaging.org.au/AMBMC/Model), and then downsampled to $50 \mu \mathrm{m}$ resolution and imported into

234 3D Slicer. Using the Editor tool at a threshold of zero, a mask was generated of the entire mouse brain,

235 and the spinal cord was manually removed using the eraser tool. The volume of the mouse brain mask

236 was calculated using the Label Statistics tool (total volume: $332.7 \mathrm{~mm}^{3}$ ). To calculate the volume of the

237 cuttlefish brain, the volume of each brain lobe in the merged, template brain was calculated using the

238 Label Statistics tool and summed (total volume: $313.7 \mathrm{~mm}^{3}$ ), see Table 2.

\section{Histological atlas}

242 Adult cuttlefish were euthanized in $10 \%$ ethanol, and the brain and connected eyes were surgically

243 removed and fixed overnight in a solution of $4 \%$ paraformaldehyde (PFA) in filtered artificial seawater

244 (FASW) at $4^{\circ} \mathrm{C}$. Note that fixing brains in a solution of PBS (instead of FASW) created abnormal cell

245 morphologies. The fixed brains were washed in PBS, the eyes were removed with a scalpel, and the

246 brains were incubated in $10 \%$ sucrose overnight followed by $30 \%$ sucrose overnight at $4^{\circ} \mathrm{C}$. The brains

247 were embedded in OCT on dry ice and stored at $-80^{\circ} \mathrm{C}$. Each brain was sliced in $100 \mu \mathrm{m}$ sections on a

248 cryostat (Leica CM3050 S), and the sections were dried overnight at room temperature. The sections

249 were stained with Phalloidin (Life Technologies \#A12379, 1/40 dilution) and NeuroTrace (Life 
250 Technologies \#N21482, 1/20 dilution) and then imaged on a custom-built Nikon AZ100 Multizoom Slide

251 Scanner. Images were registered using BrainJ (http://github.com/lahammond/BrainJ). Brightness and

252 contrast were adjusted uniformly across each image, and surrounding tissue was removed manually

253 from the image in FIJl and Photoshop. The data was manually segmented in 3D Slicer by two annotators

254 using a neuroanatomical study (Boycott, 1961) and our 3D brain atlas for reference.

\section{Whole body atlas}

258 An adult male cuttlefish was anesthetized in $\mathrm{MgCl}_{2}(17.5 \mathrm{~g} / \mathrm{L})$ and then euthanized in $10 \%$ ethanol, fixed

259 for 2 days in $4 \%$ PFA/FASW at $4^{\circ} \mathrm{C}$, and then transferred to $0.2 \%$ OMNISCAN (gadodiamide) for 2 days

260 at $4^{\circ} \mathrm{C}$ to increase contrast. The fixed specimen was suspended in fomblin in a custom-made vessel and

261 imaged on a Bruker BioSpec 94/30 horizontal small animal MRI scanner (field strength, 9.4 T; bore size,

$26230 \mathrm{~cm}$ ) equipped with a CryoProbe and ParaVision 6.0.1 software (Bruker). A 112/86-mm 1H circularly

263 polarized transmit/receive-capable volume coil was used for imaging. T1-weighted images were

264 acquired with a FLASH sequence $\left(T R=55 \mathrm{~ms}\right.$, TE $=17 \mathrm{~ms}$, FOV $=90 \times 48 \times 38 \mathrm{~mm}^{3}$, voxel size $=100 \times$

$265100 \times 100 \mu \mathrm{m}^{3}$, scan time $=2 \mathrm{hr} 47 \mathrm{~m}$ ). The scan underwent N4 bias field correction (Tustison et al.,

266 2010) and was manually segmented in 3D Slicer using anatomical descriptions (Gestal et al., 2019).

\section{Cuttlebase}

270 The Cuttlebase web content is delivered using React, a Javascript front-end framework for dynamic

271 websites. React-three-fiber (a Three.js wrapper for React) is used to assist with interactions in the 3D

272 view.

\section{3D brain}

275 In 3D Slicer, each segment (brain lobe or tract) of the final, template brain was exported as an STL file 276 and then labelled with a unique identifier in Blender, a 3D authoring software. The 3D model was 
277 exported as a GLB, and then imported into a webpage using Three.js - a Javascript library for handling

$2783 \mathrm{D}$ content on the web. A custom web-interface was created to assign colors to each of the region

279 meshes, and this data was exported as a JSON file.

\section{Histology}

282 To convert the histological annotations to high-resolution images that could be toggled on Cuttlebase,

283 each segment (brain lobe or tract) for each brain (horizontal, sagittal and transverse) was converted to a

284 binary label map in 3D Slicer and saved as a TIFF stack. Each TIFF stack was resized to the canvas size

285 of the original image in Fiji (Schindelin et al., 2012), and saved as a JPEG Stack in monochrome. The

286 images were then inverted and processed with Potrace (through a custom Node.js script), to create

287 SVGs for each region of each layer. The SVGs for all regions in a single layer were combined, and each

288 region was assigned the color corresponding to the 3D atlas. These images, along with the originals,

289 were resized and cropped for more efficient web delivery. Additionally, the cartilage in each Phalloidin

290 section was isolated (by outlining) using Adobe lllustrator, and exported as a PNG. These images were

291 used as masks on the NeuroTrace layers with the command-line tool ImageMagick, to automate this

292 process for the remaining images. Custom Bash scripts were used to manage and organize the large

293 amounts of data and the processing steps required.

295 Data availability

297 All data generated in this study is available to download from cuttlebase.org/downloads.

\section{Acknowledgements}

301 We wish to thank Connor Gibbons, Sonia Thomas, Telicia Lewis, Sarah Wilson and Josh Barber for

302 cuttlefish care, the Zuckerman Institute Cellular Imaging platform for instrument use and technical

303 support and Lokke Highstein for IT support. This project was funded by a Zuckerman Institute MRI Seed 
Grant (T.G.M. and R.A.), the Howard Hughes Medical Institute Hanna H. Gray Fellowship (T.G.M.), the

\section{Competing interests}

The authors have no competing interests.

\section{$311 \quad$ Rich media file legends}

313 Video 1. Dwarf cuttlefish produce waves (of unknown function) on their skin.

314 Video 2. Dwarf cuttlefish can walk bipedally using their ventral arms.

\section{References}

Abbott, N. J., Williamson, R., \& Maddock, L. (1995). Cephalopod Neurobiology. Oxford University Press, USA. http://books.google.com/books?id=r1MXAQAAIAAJ\&hl=\&source=gbs_api Albertin, C. B., \& Simakov, O. (2020). Cephalopod Biology: At the Intersection Between Genomic and Organismal Novelties. Annu Rev Anim Biosci, 8, 71-90. https://doi.org/10.1146/annurev-animal-021419083609

Amodio, P., Boeckle, M., Schnell, A. K., Ostojíc, L., Fiorito, G., \& Clayton, N. S. (2019). Grow Smart and Die Young: Why Did Cephalopods Evolve Intelligence. Trends Ecol Evol, 34(1), 45-56. https://doi.org/10.1016/j.tree.2018.10.010

Avants, B. B., Tustison, N. J., Song, G., Cook, P. A., Klein, A., \& Gee, J. C. (2011). A reproducible evaluation of ANTs similarity metric performance in brain image registration. Neuroimage, 54(3), 20332044. https://doi.org/10.1016/j.neuroimage.2010.09.025

Basil, J. A., Hanlon, R. T., Sheikh, S. I., \& Atema, J. (2000). Three-dimensional odor tracking by Nautilus pompilius. J Exp Biol, 203(Pt 9), 1409-1414. https://pubmed.ncbi.nlm.nih.gov/10751156 Boycott, B. B. (1961). The functional organization of the brain of the cuttlefish Sepia officinalis. Proceedings of the Royal Society of London. Series B, Biological Sciences, 153, 503-534. Boycott, B. B., \& Young, J. Z. (1957). Effects of interference with the vertical lobe on visual discriminations in Octopus vulgaris Lamarck. Proc R Soc Lond B Biol Sci, 146(925), 439-459. https://doi.org/10.1098/rspb.1957.0023

Boycott, B. B., \& Young, J. Z. (1955). A memory system in Octopus vulgaris Lamarck. Proc R Soc Lond B Biol Sci, 143(913), 449-480. https://doi.org/10.1098/rspb.1955.0024

Budelmann, B. U. (1995). The cephalopod nervous system: what evolution has made of the molluscan design. In The nervous systems of invertebrates: An evolutionary and comparative approach (pp. 115138). Springer. https://link.springer.com/chapter/10.1007/978-3-0348-9219-3_7 
Bullock, T. H., \& Budelmann, B. U. (1991). Sensory evoked potentials in unanesthetized unrestrained cuttlefish: a new preparation for brain physiology in cephalopods. J Comp Physiol A, 168(1), 141-150. https://doi.org/10.1007/BF00217112 Chichery, M. P., \& Chichery, R. (1987). The anterior basal lobe and control of prey-capture in the cuttlefish (Sepia officinalis). Physiol Behav, 40(3), 329-336. https://doi.org/10.1016/0031-9384(87)900552 Chichery, R., \& Chanelet, J. (1976). Motor and behavioral responses obtained by stimulation with chronic electrodes of the optic lobe of Sepia officinalis. Brain Res, 105(3), 525-532. https://doi.org/10.1016/0006-8993(76)90598-9 of the Squid Brain. iScience, 23(1), 100816. https://doi.org/10.1016/j.isci.2019.100816

Chung, W. S., Kurniawan, N. D., \& Marshall, N. J. (2021). Comparative brain structure and visual processing in octopus from different habitats. Curr Biol, S0960-9822(21)01532.

https://doi.org/10.1016/j.cub.2021.10.070

Crook, R. J., \& Basil, J. A. (2008). A role for nautilus in studies of the evolution of brain and behavior. Commun Integr Biol, 1(1), 18-19. https://doi.org/10.4161/cib.1.1.6465

Dietl, M. J. (1878). Untersuchungen über die Organisation des Gehirns wirbelloser Thiere (Cephalopoden, Tethys). Sitzungsberichte der Akademie der Wissenschaften in Wien, 77, 481-533. Dubas, F., Hanlon, R. T., Ferguson, G. P., \& Pinsker, H. M. (1986a). Localization and stimulation of chromatophore motoneurones in the brain of the squid, Lolliguncula brevis. J Exp Biol, 121, 1-25. https://pubmed.ncbi.nlm.nih.gov/3958673 Dubas, F., Leonard, R. B., \& Hanlon, R. T. (1986b). Chromatophore motoneurons in the brain of the squid, Lolliguncula brevis: an HRP study. Brain Res, 374(1), 21-29. https://doi.org/10.1016/00068993(86)90390-2

Fedorov, A., Beichel, R., Kalpathy-Cramer, J., Finet, J., Fillion-Robin, J. C., Pujol, S., Bauer, C., Jennings, D., Fennessy, F., Sonka, M., Buatti, J., Aylward, S., Miller, J. V., Pieper, S., \& Kikinis, R. (2012). $3 \mathrm{D}$ Slicer as an image computing platform for the Quantitative Imaging Network. Magn Reson Imaging, 30(9), 1323-1341. https://doi.org/10.1016/j.mri.2012.05.001

Fiorito, G., \& Chichery, R. (1995). Lesions of the vertical lobe impair visual discrimination learning by observation in Octopus vulgaris. Neurosci Lett, 192(2), 117-120. https://doi.org/10.1016/03043940(95)11631-6

Gaston, M. R., \& Tublitz, N. J. (2004). Peripheral innervation patterns and central distribution of fin chromatophore motoneurons in the cuttlefish Sepia officinalis. J Exp Biol, 207(Pt 17), 3089-3098. https://doi.org/10.1242/jeb.01145 Gaston, M. R., \& Tublitz, N. J. (2006). Central distribution and three-dimensional arrangement of fin chromatophore motoneurons in the cuttlefish Sepia officinalis. Invert Neurosci, 6(2), 81-93. https://doi.org/10.1007/s10158-006-0021-3 Gestal, C., Pascual, S., Guerra, Á., Fiorito, G., \& Vieites, J. M. (2019). Handbook of Pathogens and Diseases in Cephalopods. Springer.

381 https://play.google.com/store/books/details?id=aEaMDwAAQBAJ\&source=gbs_api (2021). DL-BET-A deep learning based tool for automatic brain extraction from structural magnetic resonance images in mice. In.

Gonzalez-Bellido, P. T., Scaros, A. T., Hanlon, R. T., \& Wardill, T. J. (2018). Neural control of dynamic 3dimensional skin papillae for cuttlefish camouflage. iScience, 1, 24-34. https://doi.org/10.1016/j.isci Graindorge, N., Alves, C., Darmaillacq, A. S., Chichery, R., Dickel, L., \& Bellanger, C. (2006). Effects of dorsal and ventral vertical lobe electrolytic lesions on spatial learning and locomotor activity in Sepia officinalis. Behav Neurosci, 120(5), 1151-1158. https://doi.org/10.1037/0735-7044.120.5.1151 Hanlon, R. (2007). Cephalopod dynamic camouflage. Curr Biol, 17(11), R400-4. https://doi.org/10.1016/j.cub.2007.03.034 Hanlon, R. T., \& Messenger, J. B. (1988). Adaptive coloration in young cuttlefish (Sepia officinalis L.): the morphology and development of body patterns and their relation to behaviour. Philosophical

394 Transactions of the Royal Society of London. B, Biological Sciences, 320(1200), 437-487.

395 https://royalsocietypublishing.org/doi/abs/10.1098/rstb.1988.0087 
396

397

398

399

400

401

402

403

404

405

406

407

408

409

410

411

412

413

414

415

416

417

418

419

420

421

422

423

424

425

426

427

428

429

430

431

432

433

434

435

436

437

438

439

440

441

442

443

444

445

446

447

448

Hanlon, R. T., \& Messenger, J. B. (2018). Cephalopod behaviour. Cambridge University Press. https://books.google.com/books?hl=en\&lr=\&id=oppPDwAAQBAJ\&oi=fnd\&pg=PR11\&dq=cephalopod+b ehaviour+messenger\&ots=C-2bL6f2Gz\&sig=-X_Wre4oCEhgA7Uwv95f8gNiNCE

Hillig, R. (1912). Das Nervensystem von Sepia officinalis L. Z. wiss. Zoo, 101, 736-800.

Hochner, B., Brown, E. R., Langella, M., Shomrat, T., \& Fiorito, G. (2003). A learning and memory area in the octopus brain manifests a vertebrate-like long-term potentiation. J Neurophysiol, 90(5), 3547-3554. https://doi.org/10.1152/jn.00645.2003

Jung, S.-H., Song, H. Y., Hyun, Y. S., Kim, Y.-C., Whang, I., Choi, T.-Y., \& Jo, S. (2018). A brain atlas of the long arm octopus, octopus minor. Experimental neurobiology, 27(4), 257.

https://www.ncbi.nlm.nih.gov/pmc/articles/PMC6120969

Koizumi, M., Shigeno, S., Mizunami, M., \& Tanaka, N. K. (2016). Three-dimensional brain atlas of pygmy squid, Idiosepius paradoxus, revealing the largest relative vertical lobe system volume among the cephalopods. J Comp Neurol, 524(10), 2142-2157. https://doi.org/10.1002/cne.23939

Liu, Y. C., Liu, T. H., Su, C. H., \& Chiao, C. C. (2017). Neural Organization of the Optic Lobe Changes Steadily from Late Embryonic Stage to Adulthood in Cuttlefish Sepia pharaonis. Front Physiol, 8, 538. https://doi.org/10.3389/fphys.2017.00538

Messenger, J. B. (1967). The effects on locomotion of lesions to the visuo-motor system in octopus. Proceedings of the Royal Society of London. Series B. Biological Sciences, 167(1008), 252-281. https://royalsocietypublishing.org/doi/abs/10.1098/rspb.1967.0026

Messenger, J. B. (1979). The nervous system of Loligo IV. The peduncle and olfactory lobes.

Philosophical Transactions of the Royal Society of London. B, Biological Sciences, 285(1008), 275-309. https://royalsocietypublishing.org/doi/abs/10.1098/rstb.1979.0007

Messenger, J. B. (2001). Cephalopod chromatophores: neurobiology and natural history. Biol Rev Camb Philos Soc, 76(4), 473-528. https://doi.org/10.1017/s1464793101005772

Montague, T. G., Rieth, I. J., \& Axel, R. (2021). Embryonic development of the camouflaging dwarf cuttlefish, Sepia bandensis. Dev Dyn. https://doi.org/10.1002/dvdy.375

Muntz, W. R. A., \& Raj, U. (1984). On the visual system of Nautilus pompilius. Journal of Experimental Biology, 109(1), 253-263. https://journals.biologists.com/jeb/article-abstract/109/1/253/4173

Nixon, M., \& Young, J. Z. (2003). The Brains and Lives of Cephalopods. Oxford University Press. http://books.google.com/books?id=BRvrtsuKc6MC\&hl=\&source=gbs_api

Ponte, G., Taite, M., Borrelli, L., Tarallo, A., Allcock, A. L., \& Fiorito, G. (2020). Cerebrotypes in Cephalopods: Brain Diversity and Its Correlation With Species Habits, Life History, and Physiological Adaptations. Front Neuroanat, 14, 565109. https://doi.org/10.3389/fnana.2020.565109

Reiter, S., Hülsdunk, P., Woo, T., Lauterbach, M. A., Eberle, J. S., Akay, L. A., Longo, A., Meier-Credo, J., Kretschmer, F., Langer, J. D., Kaschube, M., \& Laurent, G. (2018). Elucidating the control and development of skin patterning in cuttlefish. Nature, 562(7727), 361-366.

https://doi.org/10.1038/s41586-018-0591-3

Robertson, J. D., Schwartz, O. M., \& Lee, P. (1993). Carbocyanine dye labeling reveals a new motor nucleus in octopus brain. J Comp Neurol, 328(4), 485-500. https://doi.org/10.1002/cne.903280404 Russell, F. S., \& Steven, G. A. (1930). The swimming of cuttlefish. Nature, 125(3163), 893-893. https://www.nature.com/articles/125893a0

Saidel, W. M. (1982). Connections of the octopus optic lobe: an HRP study. J Comp Neurol, 206(4), 346358. https://doi.org/10.1002/cne.902060403

Sanders, F. K., \& Young, J. Z. (1940). Learning and other functions of the higher nervous centres of Sepia. Journal of Neurophysiology, 3(6), 501-526.

https://journals.physiology.org/doi/pdf/10.1152/jn.1940.3.6.501

Schindelin, J., Arganda-Carreras, I., Frise, E., Kaynig, V., Longair, M., Pietzsch, T., Preibisch, S., Rueden, C., Saalfeld, S., Schmid, B., Tinevez, J. Y., White, D. J., Hartenstein, V., Eliceiri, K., Tomancak, P., \& Cardona, A. (2012). Fiji: an open-source platform for biological-image analysis. Nat Methods, 9(7), 676682. https://doi.org/10.1038/nmeth.2019

Shinomiya, K., Horne, J. A., McLin, S., Wiederman, M., Nern, A., Plaza, S. M., \& Meinertzhagen, I. A. (2019). The Organization of the Second Optic Chiasm of the Drosophila Optic Lobe. Front Neural Circuits, 13, 65. https://doi.org/10.3389/fncir.2019.00065 
Shomrat, T., Graindorge, N., Bellanger, C., Fiorito, G., Loewenstein, Y., \& Hochner, B. (2011). Alternative sites of synaptic plasticity in two homologous "fan-out fan-in" learning and memory networks. Curr Biol, 21, 1773-1782. https://doi.org/10.1016/j.cub.2011.09.011

Turchetti-Maia, A., Shomrat, T., \& Hochner, B. (2019). The Vertical Lobe of Cephalopods., 558-574. https://doi.org/10.1093/oxfordhb/9780190456757.013.29 Tustison, N. J., Avants, B. B., Cook, P. A., Zheng, Y., Egan, A., Yushkevich, P. A., \& Gee, J. C. (2010). N4ITK: improved N3 bias correction. IEEE Trans Med Imaging, 29(6), 1310-1320. https://doi.org/10.1109/TMl.2010.2046908 Wells, M. J., \& Young, J. Z. (1975). The subfrontal lobe and touch learning in the octopus. Brain Res, 92(1), 103-121. https://doi.org/10.1016/0006-8993(75)90530-2 Young, J. Z. (1962). The optic lobes of Octopus vulgaris. Philosophical Transactions of the Royal Society of London. Series B, Biological Sciences, 245(718), 19-58. https://royalsocietypublishing.org/doi/pdf/10.1098/rstb.1962.0005 Young, J. Z. (1965). The central nervous system of Nautilus. Philosophical Transactions of the Royal Society of London. Series B, Biological Sciences, 249(754), 1-25. https://royalsocietypublishing.org/doi/abs/10.1098/rstb.1965.0006 Young, J. Z. (1971). The Anatomy of the Nervous System of Octopus Vulgaris. Oxford University Press, USA. http://books.google.com/books?id=rRUWAQAAIAAJ\&hl=\&source=gbs_api Young, J. Z. (1972). The organization of a cephalopod ganglion. Philos Trans R Soc Lond B Biol Sci, 263(854), 409-429. https://doi.org/10.1098/rstb.1972.0005

469 Young, J. Z. (1974). The central nervous system of Loligo. I. The optic lobe. Philos Trans R Soc Lond B Biol Sci, 267(885), 263-302. https://doi.org/10.1098/rstb.1974.0002 Young, J. Z. (1976). The nervous system of Loligo. II. Suboesophageal centres. Philos Trans R Soc Lond B Biol Sci, 274(930), 101-167. https://doi.org/10.1098/rstb.1976.0041 Young, J. Z. (1977). The nervous system of Loligo, III. Higher motor centres: the basal supraoesophageal lobes. Philosophical Transactions of the Royal Society of London. B, Biological Sciences, 276(948), 351398. https://royalsocietypublishing.org/doi/abs/10.1098/rstb.1977.0003

Young, J. Z. (1979). The nervous system of Loligo. V. The vertical lobe complex. Philosophical Transactions of the Royal Society of London. B, Biological Sciences, 285(1009), 311-354. https://royalsocietypublishing.org/doi/abs/10.1098/rstb.1979.0008 Zullo, L., Sumbre, G., Agnisola, C., Flash, T., \& Hochner, B. (2009). Nonsomatotopic organization of the higher motor centers in octopus. Curr Biol, 19, 1632-1636. https://doi.org/10.1016/j.cub.2009.07.067 
bioRxiv preprint doi: https://doi.org/10.1101/2022.01.23.477393; this version posted January 24,2022 . The copyright holder for this preprint

(which was not certified by peer review) is the author/funder, who has granted bioRxiv a license to display the preprint in perpetuity. It is made available under aCC-BY 4.0 International license.

\begin{tabular}{|c|c|c|}
\hline Brain region & Abbreviation & Function \\
\hline \multicolumn{3}{|l|}{ Supraesophageal mass } \\
\hline Vertical lobe complex & & Learning and memory (Young, 1991) \\
\hline Vertical lobe & V & \\
\hline Subvertical lobe & SV & \\
\hline Superior frontal lobe & SF & \\
\hline Inferior frontal lobe & IF & \\
\hline Posterior frontal lobe & PF & \\
\hline Basal lobe complex & & Higher motor control (Boycott, 1961) \\
\hline Anterior anterior basal lobe & AAB & Movement of head, arms and eyes \\
\hline Anterior posterior basal lobe & APB & \\
\hline Precommissural lobe & PC & \\
\hline Dorsal basal lobe & DB & \\
\hline Interbasal lobes & IB & Movement of feeding tentacles \\
\hline Median basal lobe & MB & $\begin{array}{l}\text { Movement of mantle and funnel during swimming and breathing; protraction } \\
\text { and retraction of head; movement of fins, movement of buccal mass, and } \\
\text { expansion and contraction of chromatophores }\end{array}$ \\
\hline Lateral basal lobes & LB & Control of chromatophores and papillae \\
\hline \multicolumn{3}{|l|}{ Subesophageal mass } \\
\hline Pedal lobe complex & & Intermediate and lower motor control of movement (Boycott, 1961) \\
\hline Anterior pedal lobe & AP & Movement of arms and tentacles \\
\hline Posterior pedal lobe & PP & Movement of funnel, fins and tentacles; head retraction \\
\hline Lateral pedal lobes & LP & Movement of eyes \\
\hline Anterior dorsal chromatophore lobes & $A D C$ & Control of chromatophores and papillae on the head and arms \\
\hline Anterior ventral chromatophore lobes & AVC & Control of chromatophores and papillae on the head and arms \\
\hline Magnocellular lobe complex & & Giant fiber response (escape movements) (Boycott, 1961) \\
\hline Dorsal magnocellular lobes & DM & \\
\hline Ventral magnocellular lobes & VM & \\
\hline Posterior magnocellular lobes & PM & \\
\hline Palliovisceral lobe complex & & Lower motor control of locomotion (Boycott, 1961) \\
\hline Palliovisceral lobe & PV & Control of escape movements and ink ejection \\
\hline Lateral ventral palliovisceral lobes & LPV & \\
\hline Fin lobes & $\mathrm{F}$ & Movement of fins \\
\hline Posterior chromatophore lobes & $\mathrm{PC}$ & Control of chromatophores and papillae on mantle, fin and visceral mass \\
\hline Dorsal vasomotor lobe & DV & \\
\hline Ventral vasomotor lobe & VV & \\
\hline Brachial lobe complex & & Motor control of arms and feeding \\
\hline Brachial lobe & $B$ & Intermediate motor control of arms \\
\hline Superior buccal lobe & SB & Biting movements of the buccal mass \\
\hline Inferior buccal lobe & IB & Biting movements of the buccal mass \\
\hline \multicolumn{3}{|l|}{ Periesophageal mass } \\
\hline \multicolumn{3}{|l|}{ Optic-tract complex } \\
\hline Optic lobes & 0 & Visual processing (Boycott, 1961) \\
\hline Peduncle lobes & $\mathrm{P}$ & Visuo-motor control (Messenger, 1967) \\
\hline Dorsolateral lobes & $\mathrm{DL}$ & \\
\hline Optic glands & $\mathrm{OG}$ & Neurosecretory center (Messenger, 1967) \\
\hline Olfactory lobes & $\mathrm{OL}$ & Unclear (Messenger, 1979) \\
\hline \multicolumn{3}{|l|}{ Nerve fibers } \\
\hline Ventral optic commissure & VOC & \\
\hline Optic to anterior basal lobe tracts & $\mathrm{OAB}$ & \\
\hline Subvertical to optic tracts & SOT & \\
\hline $\begin{array}{l}\text { Lateral basal to posterior chromatophore } \\
\text { lobe tracts }\end{array}$ & LBPC & \\
\hline Brachio-palliovisceral connectives & BPC & \\
\hline Anterior magnocellular commissure & AMC & \\
\hline Pallial nerves & PN & \\
\hline Optic to vertical lobe tracts & OV & \\
\hline Optic to dorsal magnocellular lobe tracts & ODM & \\
\hline
\end{tabular}

\section{Table 1. The lobes of the cuttlefish brain.}


bioRxiv preprint doi: https://doi.org/10.1101/2022.01.23.477393; this version posted January 24, 2022. The copyright holder for this preprint

(which was not certified by peer review) is the author/funder, who has granted bioRxiv a license to display the preprint in perpetuity. It is made available under aCC-BY 4.0 International license.

\begin{tabular}{|c|c|c|c|c|c|}
\hline \multirow{3}{*}{\begin{tabular}{|l} 
Abbreviation \\
OI \\
Or \\
\end{tabular}} & \multirow{3}{*}{\begin{tabular}{|l} 
Brain lobe \\
optic
\end{tabular}} & \multirow{2}{*}{$\begin{array}{r}\text { Number of voxels } \\
923066\end{array}$} & \multirow{2}{*}{\begin{tabular}{r|} 
Volume $\mathbf{m m}^{\wedge} \mathbf{3}$ \\
115.3832552 \\
\end{tabular}} & \multicolumn{2}{|c|}{ Proportion of the brain } \\
\hline & & & & $36.79 \%$ & \\
\hline & & 942863 & 117.8578803 & $37.57 \%$ & $74.36 \%$ \\
\hline $\mathrm{PI}$ & \multirow{2}{*}{ peduncle } & 6915 & 0.864375039 & $0.28 \%$ & \\
\hline $\mathrm{Pr}$ & & 6869 & 0.858625038 & $0.27 \%$ & $0.55 \%$ \\
\hline $\mathrm{OLI}$ & \multirow{2}{*}{ olfactory } & 1122 & 0.140250006 & $0.04 \%$ & \\
\hline $\mathrm{OLr}$ & & 927 & 0.115875005 & $0.04 \%$ & $0.08 \%$ \\
\hline $\mathrm{DLI}$ & \multirow{2}{*}{ dorsolateral } & 1738 & 0.21725001 & $0.07 \%$ & \\
\hline $\mathrm{DLr}$ & & 1814 & 0.22675001 & $0.07 \%$ & $0.14 \%$ \\
\hline SV & subvertical & 36379 & 4.547375203 & $1.45 \%$ & $1.45 \%$ \\
\hline $\mathrm{V}$ & vertical & 128404 & 16.05050072 & $5.12 \%$ & $5.12 \%$ \\
\hline $\mathrm{PC}$ & precommissural & 12129 & 1.516125068 & $0.48 \%$ & $0.48 \%$ \\
\hline $\mathrm{MB}$ & median basal & 16639 & 2.079875093 & $0.66 \%$ & $0.66 \%$ \\
\hline DB & dorsal basal & 43502 & 5.437750243 & $1.73 \%$ & $1.73 \%$ \\
\hline LBI & \multirow{2}{*}{ lateral basal } & 4871 & 0.608875027 & $0.19 \%$ & \\
\hline $\mathrm{LBr}$ & & 4953 & 0.619125028 & $0.20 \%$ & $0.39 \%$ \\
\hline $\mid \mathrm{BI}$ & \multirow{2}{*}{ interbasal } & 1138 & 0.142250006 & $0.05 \%$ & \\
\hline $\mathrm{IBr}$ & & 1550 & 0.193750009 & $0.06 \%$ & $0.11 \%$ \\
\hline SF & superior frontal & 26603 & 3.325375149 & $1.06 \%$ & $1.06 \%$ \\
\hline IF & inferior frontal & 5391 & 0.67387503 & $0.21 \%$ & $0.21 \%$ \\
\hline $\mathrm{PF}$ & posterior frontal & 5116 & 0.639500029 & $0.20 \%$ & $0.20 \%$ \\
\hline $\mathrm{AAB}$ & anterior anterior basal & 20829 & 2.603625116 & $0.83 \%$ & $0.83 \%$ \\
\hline APB & anterior posterior basal & 12924 & 1.615500072 & $0.52 \%$ & $0.52 \%$ \\
\hline DMI & \multirow{2}{*}{ dorsal magnocellular } & 13652 & 1.706500076 & $0.54 \%$ & \\
\hline $\mathrm{DMr}$ & & 14622 & 1.827750082 & $0.58 \%$ & $1.13 \%$ \\
\hline $\mathrm{VMI}$ & \multirow{2}{*}{ ventral magnocellular } & 2850 & 0.356250016 & $0.11 \%$ & \\
\hline $\mathrm{VMr}$ & & 2702 & 0.337750015 & $0.11 \%$ & $0.22 \%$ \\
\hline PMI & \multirow{2}{*}{ posterior magnocellular } & 7179 & 0.89737504 & $0.29 \%$ & \\
\hline PMr & & 7338 & 0.917250041 & $0.29 \%$ & $0.58 \%$ \\
\hline$B$ & brachial & 45948 & 5.743500257 & $1.83 \%$ & $1.83 \%$ \\
\hline $\mathrm{ADCl}$ & anterior dorsal chromatonhore & 790 & 0.098750004 & $0.03 \%$ & \\
\hline $\mathrm{ADCr}$ & & 747 & 0.093375004 & $0.03 \%$ & $0.06 \%$ \\
\hline $\mathrm{AVCl}$ & anterior ventral chromatonhore & 971 & 0.121375005 & $0.04 \%$ & \\
\hline $\mathrm{AVCr}$ & - & 934 & 0.116750005 & $0.04 \%$ & $0.08 \%$ \\
\hline $\mathrm{PCl}$ & nosterior chromatonhore & 3723 & 0.465375021 & $0.15 \%$ & \\
\hline $\mathrm{PCr}$ & posterior cnromatopnore & 4196 & 0.524500023 & $0.17 \%$ & $0.32 \%$ \\
\hline $\mathrm{AP}$ & anterior pedal & 31888 & 3.986000178 & $1.27 \%$ & $1.27 \%$ \\
\hline $\mathrm{PP}$ & posterior pedal & 30382 & 3.79775017 & $1.21 \%$ & $1.21 \%$ \\
\hline LPI & Iateral nedal & 7817 & 0.977125044 & $0.31 \%$ & \\
\hline $\mathrm{LPr}$ & flateral pedal & 8302 & 1.037750046 & $0.33 \%$ & $0.64 \%$ \\
\hline PV & palliovisceral & 27149 & 3.393625152 & $1.08 \%$ & $1.08 \%$ \\
\hline LPVI & lateral nallinvisceral & 5252 & 0.656500029 & $0.21 \%$ & \\
\hline LPVr & flateral palliovisceral & 4226 & 0.528250024 & $0.17 \%$ & $0.38 \%$ \\
\hline $\mathrm{Fl}$ & fin & 11736 & 1.467000066 & $0.47 \%$ & \\
\hline $\mathrm{Fr}$ & & 11120 & 1.390000062 & $0.44 \%$ & $0.91 \%$ \\
\hline OGI & ontic aland 120 & 434 & 0.054250002 & $0.02 \%$ & \\
\hline $\mathrm{OGr}$ & Joptic giandu & 744 & 0.093000004 & $0.03 \%$ & $0.05 \%$ \\
\hline $\mathrm{VV}$ & ventral vasomotor & 3946 & 0.493250022 & $0.16 \%$ & $0.16 \%$ \\
\hline DV & dorsal vasomotor & 6353 & 0.794125036 & $0.25 \%$ & $0.25 \%$ \\
\hline VOC & ventral optic commissure & 4721 & 0.590125026 & $0.19 \%$ & $0.19 \%$ \\
\hline LBPCl & latera h hal to necterior chromaton hore tract & 807 & 0.100875005 & $0.03 \%$ & \\
\hline LBPCr & flateral dasal to posterior cnromatopno tract & 897 & 0.112125005 & $0.04 \%$ & $0.07 \%$ \\
\hline $\mathrm{BPCl}$ & 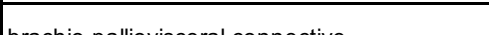 & 2908 & 0.363500016 & $0.12 \%$ & \\
\hline $\mathrm{BPCr}$ & brachio-palliovisceral connective & 3143 & 0.392875018 & $0.13 \%$ & $0.24 \%$ \\
\hline $\mathrm{PNI}$ & nallial nerve & 2437 & 0.304625014 & $0.10 \%$ & \\
\hline $\mathrm{PNr}$ & & 2324 & 0.290500013 & $0.09 \%$ & $0.19 \%$ \\
\hline AMC & anterior magnocellular commissure & 1852 & 0.23150001 & $0.07 \%$ & $0.07 \%$ \\
\hline SOTI & subvertical to ontic tracts & 964 & 0.120500005 & $0.04 \%$ & \\
\hline SOTr & subvertical to optic tracts & 660 & 0.082500004 & $0.03 \%$ & $0.06 \%$ \\
\hline $\mathrm{OABI}$ & ontic to anterior hasal lohe tract & 5414 & 0.67675003 & $0.22 \%$ & \\
\hline $\mathrm{OABr}$ & & 4805 & 0.600625027 & $0.19 \%$ & $0.41 \%$ \\
\hline $\mathrm{OVI}$ & ontic to vertical lohe tract & 7072 & 0.88400004 & $0.28 \%$ & \\
\hline $\mathrm{OVr}$ & 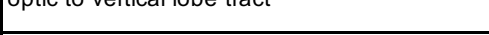 & 8156 & 1.019500046 & $0.33 \%$ & $0.61 \%$ \\
\hline ODMI & ontic to dorsal magnocellubar lohe tract & 1259 & 0.157375007 & $0.05 \%$ & \\
\hline ODMr & Optic to dorsal magnocelluar lobe tract & 1174 & 0.146750007 & $0.05 \%$ & $0.10 \%$ \\
\hline Entire brain & & 2509336 & 313.667014 & $100.00 \%$ & $100.00 \%$ \\
\hline
\end{tabular}

Table 2. Volume of cuttlefish brain lobes 
bioRxiv preprint doi: https://doi.org/10.1101/2022.01.23.477393; this version posted January 24, 2022. The copyright holder for this preprint (which was not certified by peer review) is the author/funder, whoh h Individual MRI scans Manual annotationvailable under a he mask 4 mprovement license. Manual correction
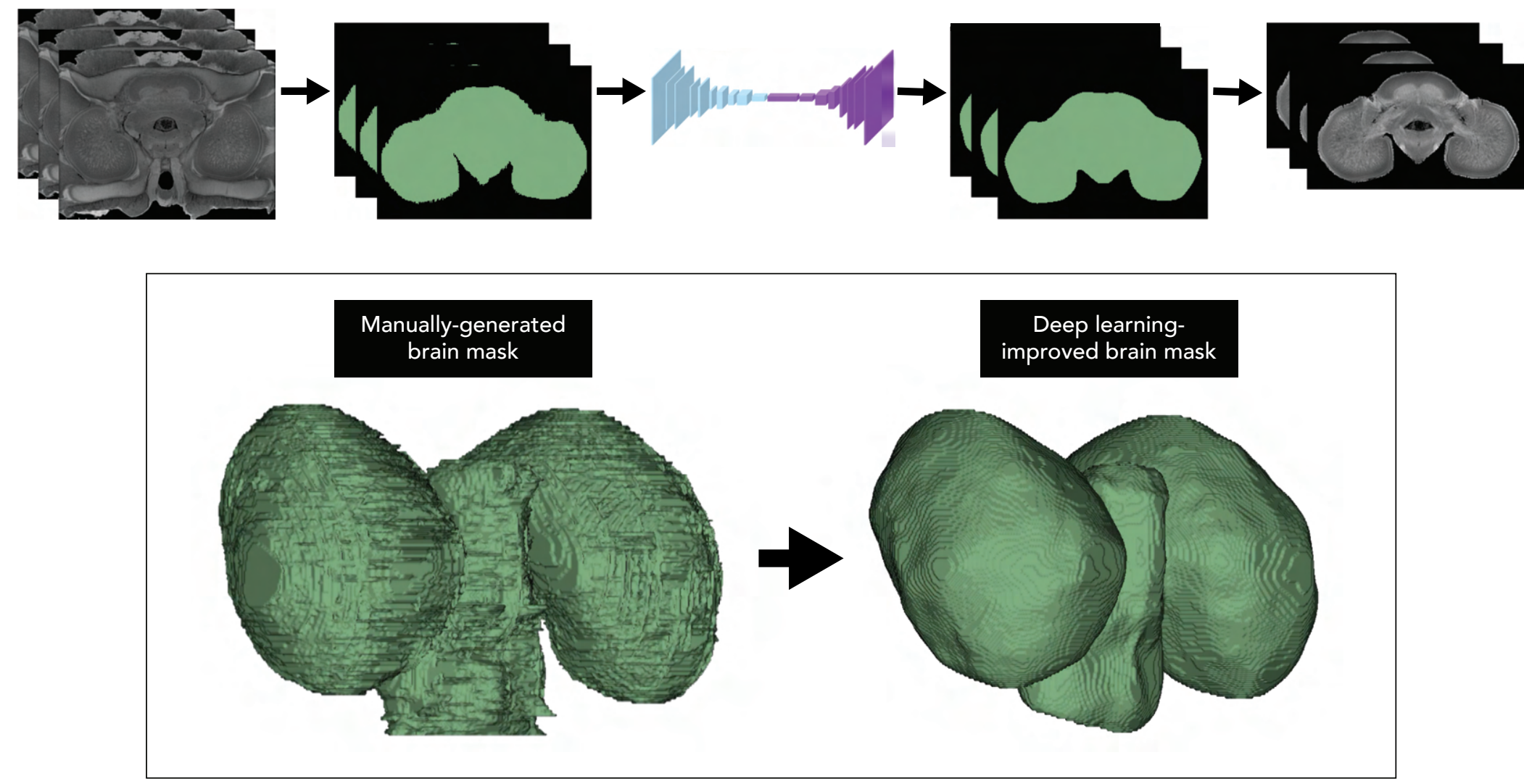

Figure S1. Deep learning pipeline for improving manually-generated brain masks. 